\title{
ANÁLISE DOS CUSTOS HOSPITALARES EM UM SERVIÇO DE EMERGÊNCIA
}

\author{
Gerardo Vasconcelos Mesquita ${ }^{1}$ Fernando Amaro Farias Veloso de Oliveira², Ana Maria Ribeiro dos Santos ${ }^{3}$, \\ Fabrício Ibiapina Tapety 4 , Maria do Carmo de Carvalho e Martins ${ }^{5}$, Carmem Milena Rodrigues Siqueira Carvalho ${ }^{6}$
}

${ }^{1}$ Doutor em Cirurgia Traumato-Ortopédica. Professor do Curso de Fisioterapia da Faculdade de Saúde, Ciências Humanas e Tecnológicas do Piauí (NOVAFAPI). Professor Adjunto Universidade Federal do Piauí (UFPI). Piauí, Brasil. E-mail: gvmesquita@uol.com.br

2 Fisioterapeuta da Clínica Corpus. Piauí, Brasil. E-mail: s.amaro.09@hotmail.com

${ }^{3}$ Mestre em Enfermagem. Professora do Curso de Graduação em Enfermagem da Faculdade NOVAFAPI. Professor Assistente do Centro de Ciências da Saúde da UFPI. Piauí, Brasil. E-mail: ana.mrsantos@gmail.com

${ }^{4}$ Doutor em Odontologia Clínica. Professor do Curso de Graduação em Odontologia NOVAFAPI. Piauí, Brasil. E-mail: ftapety@ novafapi.com.br

${ }^{5}$ Doutora em Ciências Biológicas. Professora do Curso de Graduação em Medicina NOVAFAPI. Piauí, Brasil. E-mail: ccarvalho@ novafapi.com.br

${ }^{6}$ Doutora em Dentística e Endodontia. Professora da Faculdade NOVAFAPI e do Centro de Ciências da Saúde UFPI. Piauí, Brasil. E-mail: ccarvalho@novafapi.com.br

RESUMO: Atualmente os acidentes e violências são classificados pelo Ministério da Saúde, conforme a Classificação Internacional de Doenças como Causas Externas. Este estudo objetivou analisar os custos hospitalares por causas externas em um Serviço de Emergência de Teresina-PI. Estudo descritivo, com abordagem quantitativa cujos dados foram coletados através do Sistema de Informações Hospitalares do Hospital Getúlio Vargas, referentes aos anos de 2005 a 2007. Os resultados mostram que no Piauí a situação tem sido semelhante ao restante do país, observando-se que em Teresina as causas externas vêm aumentando o número de ocorrências e elevando o custo das admissões hospitalares. Constatou-se crescente aumento das admissões (19,5\%) e dos custos hospitalares (114,9\%) decorrentes destas causas. Conclui-se que estes dados podem subsidiar a implementação de políticas públicas que previnam e combatam esse problema, como também sensibilizar os profissionais de saúde, haja vista a grande demanda de vítimas por essas causas, exigindo profissionais qualificados.

DESCRITORES: Causas externas. Violência. Acidentes. Custos hospitalares.

\section{ANALYSIS OF THE HOSPITAL COST IN AN EMERGENCY SERVICE}

\begin{abstract}
Currently, accidents and violence are classified in Brazil by the Ministry of Health, according to the International Classification of Diseases as External Causes. The objective of this study is to analyze hospital costs resulting from external causes at the Emergency Ward in Teresina-PI, Brazil. The data was collected through the Hospital Information System at Getúlio Vargas Hospital from 2005 to 2007. The results presented in show that the situation in Teresina, Piauí has been similar to the rest of the Brazil, given that in Teresina, external causes have been increasing in number of occurrences and has elevated the cost of hospital admissions. An increase in admissions (19.5\%) and hospital costs (114.9\%) because of these causes was observed. One concludes that this data may aid in the implementation of public policies which prevent and combat this problem, as well as sensitize health care professionals, since a great number of the external cause victims require qualified professional assistance.
\end{abstract}

DESCRIPTORS: External causes. Violence. Accidents. Hospital costs.

\section{ANALISIS DE LOS COSTOS HOSPITALARES EN UN SERVICIO DE EMERGENCIA}

RESUMEN: Actualmente los accidentes y violencia son clasificados por el Ministerio de la Salud, de acuerdo con la Clasificación Internacional de Enfermedades como Causas Externas. El presente es un estudio descriptivo, con abordaje cuantitativo, cuyo objetivo fue analizar los costos hospitalares por causas externas en un Servicio de Emergencia en Teresina-PI. Los datos fueron recogidos a través del Sistema de Informaciones Hospitalares del Hospital Getulio Vargas, referentes a los años de 2005 a 2007. Los resultados presentados muestran que en Piauí la situación ha sido semejante al resto del país, observándose que en Teresina las causas externas están aumentando el número de ocurrencias y elevando el costo de las admisiones hospitalares. Se constató un creciente aumento de las admisiones $(19,5 \%)$ y de los costos hospitalares $(114,9 \%)$ debido a estas causas. Se puede concluir que estos datos pueden ayudar a la implementación de políticas públicas que prevengan y combatan ese problema, como también sensibilizar los profesionales del área de la salud, pues, la gran demanda de víctimas de causas externas, exige profesionales calificados.

DESCRIPTORES: Causas externas. Violencia. Accidentes. Costos de hospital. 


\section{INTRODUÇÃO}

Os acidentes e violências representam um desafio para a saúde pública, tanto no seu combate e controle, quanto no planejamento de estratégias preventivas visando à redução de suas ocorrências que causam vários danos à população, dentre eles, os sociais, psicológicos e físicos, abrangendo altos índices de morbimortalidade e elevados custos hospitalares decorrentes do tratamento e reabilitação de suas vítimas. A violência apesar de ter conceito amplo, complexo e polissêmico, pode ser definida de forma genérica como ações realizadas por indivíduos, grupos, classes ou nações que provocam danos físicos ou morais a si próprios ou a outros, embora não seja um evento de responsabilidade da saúde, gera sérios efeitos nesta área. ${ }^{1}$

Os acidentes e as violências configuram, assim, um conjunto de agravos à saúde que podem ou não induzir a vítima ao óbito, no qual estão inseridas as causas ditas acidentais - geradas pelo trânsito, trabalho, quedas, envenenamentos, afogamentos e outras formas de acidentes - e as causas intencionais (agressões e lesões autoprovocadas). Esse conjunto de agravos consta na Classificação Internacional de Doenças (CID) sob a denominação de causas externas. ${ }^{2}$

A Organização Pan-Americana da Saúde em seu documento sobre o tema destaca que a violência é reconhecidamente um problema social e de saúde pública em vários países do mundo e alerta sobre a sobrecarga que suas lesões e seqüelas impõem aos serviços de saúde em decorrência das necessidades de cuidados especializados que suas vítimas apresentam. ${ }^{3}$

As lesões traumáticas são um dos maiores problemas de saúde pública no mundo. Nos Estados Unidos, tais ferimentos causam cerca de 150.000 mortes anualmente, com 31 milhões de ferimentos não fatais nos setores de emergência. Assim, estes setores representam uma relevante fonte de informação referente a tais lesões. ${ }^{4}$

O banco de dados do Major Trauma Outcome Study dos Estados Unidos é reconhecido como o de maior impacto na análise da morbidade hospitalar por trauma e, seus estudos têm sido vitais para a avaliação da gravidade e dos atendimentos nesta especialidade. $^{5}$

A área da saúde pública tem, aos poucos, direcionado suas ações no intuito de atender os efeitos da violência e também de promover a sua prevenção. Do ponto de vista econômico, a violência representa um custo difícil de ser mensurado, porém, elevado, visto que afeta principalmente uma faixa etária populacional produtiva que é arrancada bruscamente do meio e da forma em que vive, seja pela morte prematura ou pela ocorrência de sequielas, na maioria das vezes, graves e irreversíveis. ${ }^{1}$ As pessoas mais jovens e os homens são os que sofrem maior ocorrência de morte por causas violentas fazendo com que a sociedade deixe de contar com a força de trabalho e a contribuição de indivíduos na faixa etária em que são mais produtivos. ${ }^{6}$

No Brasil, vem ocorrendo um importante crescimento das causas externas, que tem alterado a morbimortalidade da população. ${ }^{5}$ Os dados revelam que o Brasil passou de 59,0 mortes por causas externas (acidentes e violências) por 100 mil habitantes na década de 1980 , para 72,5 em 2002. ${ }^{2-7}$

Tais causas conferem ônus econômicos e sociais elevados, incluindo custos hospitalares. As internações por causas externas tendem a ser mais caras do que a média das hospitalizações pagas pelo Sistema Único de Saúde (SUS). ${ }^{5}$ Assim, enquanto o valor pago, em média, por cada internação de causa natural corresponde a $\mathrm{R} \$ 239,40$, as hospitalizações por causas externas custam, em média, cerca de $37 \%$ a mais, ou seja $\mathrm{R} \$ 328,785 .{ }^{8-9}$

Verifica-se, assim, que os acidentes e as violências são dois grandes responsáveis pelos atendimentos na saúde pública. Embora, atualmente, existam muitas pesquisas referentes ao assunto, somente algumas delas são direcionadas para a organização dos serviços e das despesas hospitalares. ${ }^{10}$

As internações oriundas de lesões geradas por causas externas nos hospitais próprios ou conveniados com o SUS no ano 2000, representaram cerca de $6 \%$ do total de hospitalizações. Esse valor parece ser baixo, porém representa algo em torno de 700 mil internações/ano. Ainda analisando os custos hospitalares, foi possível observar que, no estado de São Paulo, esse valor equivale a algo próximo de $8 \%$ do total despendido, o que deixa claro que o paciente traumatizado é mais oneroso, com gasto/dia $60 \%$ mais elevado em relação a pacientes internados devido a causas naturais. ${ }^{1}$

No Brasil, em 2005, foram realizadas 11.429.133 internações em hospitais próprios ou conveniados do SUS. As lesões e envenenamentos decorrentes de acidentes e violências foram responsáveis por 786.768 internações $(6,8 \%)$, ocupando o $6^{\circ}$ lugar no perfil de morbidade geral da população do país. ${ }^{7}$

No Nordeste, o custo médio de uma internação por acidente e violência representa para o SUS $89 \%$ a mais do que o custo médio das demais inter- 
nações, enquanto no país, essa diferença gira em torno de $37 \% .{ }^{8}$ A cidade de Teresina possui elevadas taxas de mortalidade por causas externas e em relação à morbidade por estas causas, apresentou em 2005, uma proporção de 6,64 internações por lesões, envenenamentos e outras causas externas, com uma taxa de 383,3 por 100 mil habitantes, correspondendo à terceira maior do Nordeste. Portanto, semelhante aos valores apresentados pela nação, de 6,68 internações, com uma taxa de 390,7 por 100 mil habitantes. Em relação a lesões corporais registradas na Polícia Civil, em 2003, foi observada uma concentração de ocorrências na capital, correspondendo a 92,2\% dos casos, quase que a totalidade das ocorrências registradas no estado do Piauí. Sobre os acidentes de trânsito registrados em Teresina, no ano de 2002, referentes a vítimas fatais e não fatais, os valores foram 138,5 e 5,4 respectivamente. Estas informações são de grande relevância no desenho das políticas públicas de todas as esferas de governo, auxiliando no planejamento das ações em saúde em qualquer nível de complexidade. ${ }^{7}$

Por todo este contexto, este estudo objetivou analisar os custos hospitalares decorrentes de causas externas em um Serviço de Emergência de Teresina-PI.

\section{METODOLOGIA}

Estudo descritivo com abordagem quantitativa, cujos dados foram coletados através do Sistema de Informações Hospitalares do Hospital Getúlio Vargas (HGV), localizado em Teresina-PI, referente ao período de janeiro de 2005 a dezembro de 2007. Foram levantadas todas as admissões realizadas no Serviço de Emergência do referido hospital, e a seguir a seleção e agrupamento dessas admissões baseando-se na CID 10.

As admissões por lesões e envenenamento abrangeram as seguintes ocorrências: acidentes de bicicleta, de motocicleta, no trabalho, de veículo estrada/ zona rural, de veículo zona urbana; agressão física; agressão física com arma branca; agressão física com arma de fogo; agressão física doméstica; atropelamento; envenenamento; espancamento; pancada no olho; perfuração do olho; queda; queimadura e queimadura com fogos de artifício.

As demais ocorrências foram reunidas no grupo denominado de outras causas, que correspondia às internações por: cansaço; choque elétrico; consulta de retorno; corpo estranho (indefinido) na garganta, no nariz, no ouvido, no olho; desmaio; diarréia; dor abdominal, de cabeça, de ouvido, lombar, na perna, no olho, no peito; epistaxe; febre; mal súbito; miíase; náusea; parecer/tratamento odontológico; urticária; vômitos. Foi constituído ainda um terceiro grupo que correspondeu às admissões sem causa especificada, chamado de causas ignoradas.

Dentro desses três grupos acima citados, foram levantados além do número de admissões, os respectivos custos hospitalares, que foram obtidos mensalmente e a seguir agrupados anualmente.

A análise descritiva dos dados foi realizada através da obtenção das distribuições absolutas e percentuais na plataforma Excel 2007, permitindo uma melhor observação e análise das variações do número de admissões e dos seus respectivos custos hospitalares, facilitando a compreensão dos resultados obtidos.

O estudo foi aprovado pelo Comitê de Ética em Pesquisa da NOVAFAPI, sob o No 017/08 e contou também com autorização da Comissão de Ética em Pesquisa da instituição na qual foram coletados os dados (HGV), atendendo aos preceitos da Resolução No 196/96 do Conselho Nacional de Saúde.

\section{RESULTADOS E DISCUSSÃO}

Os traumas e lesões oriundos das causas externas são um problema grave no Brasil, e em Teresina foram responsáveis pelo aumento do número de admissões, bem como por grande parte dos custos hospitalares no HGV, que é o principal serviço de referência em trauma da região. É um sério problema que afeta também a área da Fisioterapia, que tem evoluído enquanto ciência, com novas tecnologias e formas de tratamento, sendo responsável pela reabilitação de vítimas, geralmente politraumatizadas, elevando custos e dificultando o tratamento.

A Tabela 1 mostra os custos hospitalares por causas externas, outras causas e causas ignoradas, nos anos de 2005, 2006 e 2007, respectivamente, evidenciando a dimensão dos custos hospitalares representados pelas causas externas em relação às demais causas de admissão.

Observa-se que no ano de 2005, os custos hospitalares por causas externas $(41,4 \%)$ se aproximam em valor percentual dos custos hospitalares por outras causas $(58,4 \%)$, verificando-se assim que as causas externas geram despesas hospitalares elevadas, evidenciando seu alto custo terapêutico. Os custos hospitalares por causas externas envol- 
vem procedimentos mais caros, maior número de cirurgias, permanência em unidade de terapia intensiva, maior consumo de medicamentos e en- volvimento multiprofissional na abordagem das vítimas, geralmente politraumatizadas, tornando assim a internação mais cara.

Tabela 1 - Custos hospitalares segundo grupo de causas no Serviço de Emergência do Hospital Getúlio Vargas. Teresina - PI, 2005 a 2007

\begin{tabular}{|c|c|c|c|c|c|c|}
\hline & \multicolumn{6}{|c|}{ Custos hospitalares } \\
\hline & \multicolumn{2}{|c|}{2005} & \multicolumn{2}{|c|}{2006} & \multicolumn{2}{|c|}{2007} \\
\hline & $\mathbf{R} \boldsymbol{\$}^{*}$ & $\%$ & $\mathbf{R} \$$ & $\%$ & $\mathbf{R} \$$ & $\%$ \\
\hline Causas externas & $232.626,91$ & 41,4 & $287.097,30$ & 49,7 & $500.031,70$ & 63,9 \\
\hline Outras causas & $327.697,75$ & 58,4 & $290.252,60$ & 50,2 & $270.962,10$ & 34,6 \\
\hline Causas ignoradas & $1.087,25$ & 0,2 & 626,88 & 0,1 & $11.468,27$ & 1,5 \\
\hline Total & $561.411,91$ & 100,0 & $577.976,78$ & 100,0 & $782.462,07$ & 100,0 \\
\hline
\end{tabular}

* Valores em reais

A literatura afirma que as internações por causas externas tendem a ser mais onerosas do que a média das hospitalizações pagas pelo SUS, embora representem uma proporção relativamente menor em relação ao total de dias de hospitalização, com tempo médio de internação de 5,2 dias, comparado com 6,1 dias para todas as outras causas. ${ }^{8} \mathrm{No} \mathrm{HGV}$, segundo indicadores mensais de 2006, a média de permanência hospitalar geral é de 5,47 dias. O maior custo médio das internações por causas externas indica que estas hospitalizações apresentam um custo/dia significativamente maior $(60 \%)$ que a média geral dos casos, correspondendo a $\mathrm{R} \$ 63,11$ comparados com $\mathrm{R} \$ 39,44$ das demais causas. Isto implica em atendimentos de casos mais agudos e que, portanto, tendem a consumir recursos de maneira mais intensiva. ${ }^{8}$ Este fato foi confirmado ao se observar nas Autorizações de Internação Hospitalar, que praticamente $70 \%$ das internações devido às causas externas foram classificadas como cirúrgicas, enquanto essa proporção foi $22,6 \%$ para o total das hospitalizações.

Comparando os custos hospitalares dos dois primeiros anos, percebe-se que em 2005 o custo hospitalar com causas externas correspondia a $41,4 \%$, passando a 49,7 em 2006. Ocorreu em contrapartida, uma redução nos custos com outras causas de 58,4\% em 2005 para 50,2\% em 2006, resultando em um equilíbrio percentual, no que diz respeito às despesas hospitalares, entre causas externas e outras causas de aproximadamente $50 \%$ para cada grupo neste último ano.
O aumento dos custos hospitalares com as causas externas no $\mathrm{HGV}$, na transição do ano de 2005 para 2006, foi atribuído ao aumento das internações por estas causas, que pode ser fruto do desconhecimento da população em relação à existência e função dos postos de saúde, responsáveis pela resolução de problemas de baixa e média complexidade ou talvez a inadequação desses postos para o atendimento, aumentando assim a demanda de pacientes para o HGV. Também, os vários anos de prestações de serviço do referido serviço e seu alto poder de resolução, talvez mantenham a credibilidade de grande parte da população que busca seu atendimento emergencial gerando esse crescente número de internações e, conseqüentemente, os elevados custos hospitalares com causas externas.

Associando-se os custos entre os dois últimos anos, constata-se aumento percentual dos custos hospitalares com causas externas em 2006, verificando-se o mesmo em 2007. No ano de 2006 o percentual correspondente aos custos com causas externas foi $49,7 \%$, passando em 2007 para $63,9 \%$, ou seja, um percentual mais elevado que o observado na transição do ano de 2005 para 2006 que foi $8 \%$. Sobre os custos hospitalares com outras causas o fenômeno também se repete, observando-se uma queda para $34,6 \%$, representando uma redução em torno de $15 \%$.

As razões que podem levar ao aumento dos custos hospitalares com as causas externas podem estar relacionadas ao aumento do consumo de materiais e medicamentos hospitalares verifica- 
dos nestas causas, assim como a variação de seus valores no mercado. A grande maioria destes produtos é de utilização constante, baixa vida útil e elevados preços de aquisição, como sondas, cateteres, drenos, fios cirúrgicos, fixadores externos, curativos, oxigênio utilizado em ventilação mecânica invasiva e não-invasiva, nutrição parenteral e outros. Estes custos podem variar, dependendo do tipo de causa externa, da magnitude do trauma e do tempo de permanência hospitalar, exigindo abordagem diferenciada.

Na Tabela 2 observa-se a quantidade de admissões por causas externas, causas ignoradas e outras causas, nos anos 2005, 2006 e 2007, evidenciando o aumento percentual das causas externas e a redução das outras causas.

Tabela 2 - Admissões segundo grupo de causas no Serviço de Emergência do Hospital Getúlio Vargas. Teresina - PI, 2005 a 2007

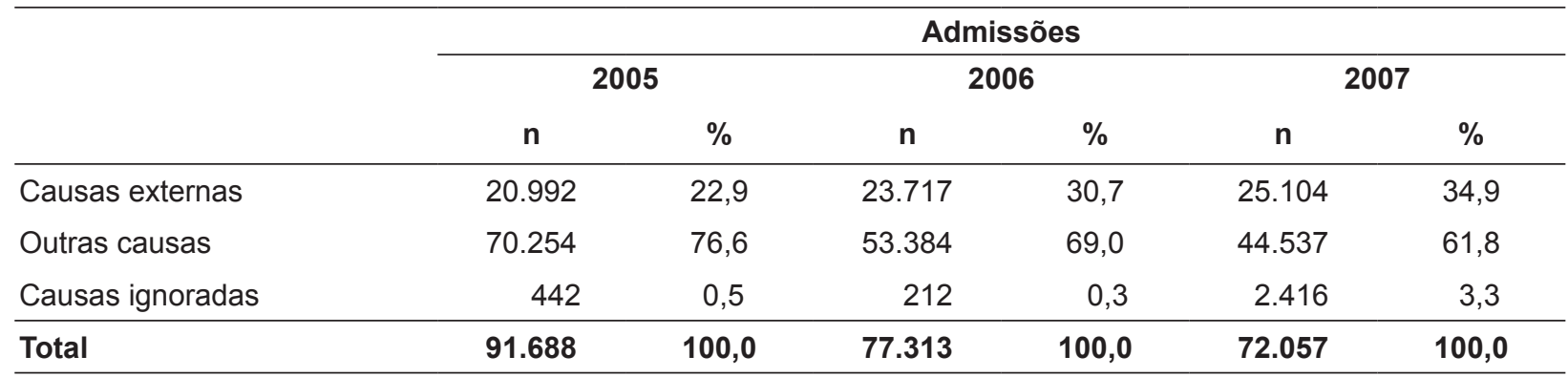

Em 2005 as admissões por causas externas corresponderam a 22,9\% e em 2006 passaram a representar 30,7\%. As outras causas apresentaram um decréscimo percentual, pois em 2005 foram $76,6 \%$ e em 2006 caíram para $69 \%$. Na transição do ano de 2006 para 2007, percebe-se novo aumento percentual das causas externas, visto que em 2006 correspondem a 30,7\% e em 2007 somam 34,9\%. Com relação às outras causas, houve decréscimo percentual na quantidade de admissões, em 2006 representaram $69 \%$ e em $2007,61,8 \%$, mantendo uma redução percentual considerada constante no decorrer dos anos. Assim, observa-se que com o aumento do número de admissões por causas externas, ocorreu redução das admissões por outras causas no HGV.

Entretanto, chama a atenção a ausência de relação entre número de admissões e custos hospitalares, visto que se observou aumento de $8 \%$ no número de admissões por causas externas do ano de 2005 para 2006 e de $4 \%$ do ano de 2006 para 2007 portanto, metade do aumento observado no período anterior. No entanto, os custos hospitalares não acompanharam essa redução. $\mathrm{Na}$ realidade observa-se um processo inverso, pois os custos hospitalares por estas causas praticamente dobraram no ano de 2006 para 2007.

Vários fatores podem ser responsáveis por este acontecimento, dentre eles: o fato de Teresina, capital do estado do Piauí ser uma metrópole em constante desenvolvimento urbano e crescimento populacional, sendo que paralelo a isso cresce também a violência e os acidentes que representam traumas mais agudos e carecem de uma abordagem intensiva e multidisciplinar o que torna a intervenção mais onerosa. Teresina apresentou em 2003, a segunda maior taxa de lesões corporais registradas na Polícia Civil do Brasil, sendo esta de $92,2 \%$, perdendo apenas para Manaus-AM. Este é um dos fatores que refletem o crescimento populacional juntamente com a carência de políticas públicas para a prevenção e combate das causas externas, dentre elas as lesões corporais, não deixando também de ser um reflexo da falta de oportunidade envolvendo desemprego e falta de acesso à educação. ${ }^{7}$

$\mathrm{Na}$ maioria das capitais das regiões metropolitanas, a participação das causas externas nas hospitalizações é cada vez mais intensa, demandando número cada vez maior de leitos hospitalares, situando-se entre a segunda e quarta causa de internação. ${ }^{7}$ No Piauí não é diferente, através destes dados evidencia-se a repercussão das causas externas, gerando custos cada vez mais elevados para o HGV, pois na grande maioria dos casos, essas vítimas necessitam de uma abordagem intensiva.

Existem algumas justificativas para tal fato, uma delas refere-se à segurança pública. Infere-se que o número de policiais tem sido insuficiente para coibir a violência na cidade, agravada pelos períodos 
de greve da Polícia Militar do Piauí. Acredita-se que os baixos salários associados às precárias condições de trabalho da PM-PI, desmotivam o trabalhador na execução do seu serviço, contribuindo para o crescimento da violência em Teresina.

Outro aspecto importante em relação ao aumento dos custos hospitalares devido a causas externas no HGV pode estar relacionado ao aumento do consumo de bebidas alcoólicas e até mesmo o uso de drogas ilícitas durante as atividades de lazer, sendo estas, talvez, as grandes responsáveis pelo aumento do número de agressões notadamente entre jovens. Esses incidentes ocorrem também em locais de grande aglomeração como shows e micaretas, dentre outros, os quais ocorrem, geralmente, nos finais de semana. Verifica-se que a maior parte desses agravos $(47,9 \%)$ ocorre as sextas, sábados e domingos, dias mais utilizados para o lazer da população, sendo os dias do meio da semana (quarta e quinta-feira) o período de menor ocorrência desses eventos. ${ }^{9}$

Além do que já foi citado como responsável pelo aumento dos custos hospitalares e das internações por causas externas, assinala-se o fato de Teresina apresentar uma grande influência na saúde da região, assistindo, aproximadamente, 5.000.000 pessoas de vários estados, tornando-se um dos pólos de referência em Saúde do Brasil, principalmente no eixo Meio-Norte. ${ }^{12} \mathrm{O}$ desenvolvimento da rede hospitalar de Teresina, e dos demais estabelecimentos de saúde, tem sido acompanhado pelo crescimento tecnológico da engenharia médico-hospitalar e dos recursos humanos em todos os níveis e profissões. ${ }^{13}$

Esses fatores desencadeiam deslocamentos de pacientes de alguns estados da federação em busca de atendimento hospitalar em Teresina, sendo grande parte atendida no HGV, vindos principalmente de estados vizinhos como o Maranhão com 53.530/ano e Ceará com 18.025/ano, correspondendo a cerca de 93.825 migrantes vindos de todo o Brasil para o Piauí. ${ }^{11}$

\section{CONCLUSÃO}

Conclui-se que as causas externas são um problema crescente e de grande repercussão social em Teresina-PI. Quanto aos custos hospitalares, os resultados mostram que as causas externas são, além de causa importante de admissão hospitalar no HGV também responsáveis por um elevado e crescente aumento de custos hospitalares no período estudado. Ressalta-se que de 2005 a 2007, no HGV, foram gastos R\$ 1.019.755,90 com admissões por causas externas, enquanto por outras causas, no mesmo período, foram despendidos $\mathrm{R} \$ 888.912,44$.

Estas informações são muito importantes no desenho das políticas públicas de todas as esferas de governo. Podem também auxiliar no planejamento das atividades de saúde, em especial, ações de promoção e prevenção, no sentido de refletir e discutir sobre as causas destes acidentes e admissões, a fim de subsidiar campanhas de prevenção, em todos os níveis de assistência e complexidade do sistema.

Embora as limitações dos dados tenham impedido um estudo mais aprofundado em termos de custos, bem como a incorporação de vários elementos particularmente importantes, espera-se que estes resultados sirvam de estímulo a novas investigações e aprofundamentos sobre o tema em nossa realidade.

\section{REFERÊNCIAS}

1. Jorge MHPM. Violência como problema de saúde pública. Ciênc Cult. 2002 Jun-Set; 54(1):52-3.

2. Ministério da Saúde (BR). Política nacional de redução da morbimortalidade por acidentes e violências: Portaria MS/GM n 737 de 16 de maio de 2001 publicada no Diário Oficial da União Nº 96 de 18 de maio de 2001 Brasília (DF): MS; 2001.

3. Krug EG, Dahlberg LL, Mercy JA, Zwi AB, Lozano R, editors. World Report on violence and health [CD-Rom]. Geneva (SW): World Health Organization; 2002.

4. Odero W, Tierney W, Einterz R, Mungai S. Using an electronic medical record system to describe injury epidemiology and health care utilization at an innercity hospital in Indiana. Inj. Control Saf Promot. 2004 Dec; 11(4):269-79.

5. Jorge MHPM, Koizumi MS. Gastos governamentais do SUS com internações hospitalares por causas externas: análise no Estado de São Paulo, 2000. Rev Bras Epidemiol. 2004 Jun; 7(2):228-38.

6. Feijó MCC, Portela MC. Variação nos custos de interações hospitalares por lesões: os casos dos traumatismos cranianos e acidentes por arma de fogo. Cad Saúde Públ. 2001 Mai-Jun; 17(3):627-37.

7. Lima LMC, Souza ER. Panorama da violência urbana do Brasil e suas capitais. Ciênc Saúde Col. 2006; 11(Supl.1): 211-22.

8. Iunes RF. Impacto econômico das causas externas no Brasil: um esforço de mensuração. Rev. Saúde Públ. 1997; 31(Supl.4):38-46.

9. Mesquita Filho M, Jorge MHPM. Características da morbidade por causas externas em serviço de urgência. Rev Bras Epidemiol. 2007 dez; 10(4):679-91. 
10. Jedrzejczyk T, Zarzeczna-Baran M, Nowalinska M. Health services provided for victims of traumas and accidents in the Gdansk metropolitan area. Wiad Lek. 2002; 55(Supl.1):178-83.

11. Instituto Brasileiro de Geografia e Estatística [página na Internet]. IBGE Cidades@. [acesso em 2008 Mai15]. Disponível em: http://www.ibge.gov.br/ cidadesat/default.php.

12. Instituto Brasileiro de Geografia e Estatística.
Teresina em dados. População migrante de Teresina segundo a unidade da federação de origem [CDRom]. Teresina (PI): Prefeitura Municipal de Teresina; 2000.

13. Sociedade Brasileira de Cardiologia [página na Internet]. Senador pretende unir governo e instituições. [acesso em 2008 Mai 15]. Disponível em: http://jornal.cardiol.br/2002 / mar-abr/paginas/ other/entrevista/default.asp
Gerardo Vasconcelos Mesquita

Rua Olavo Bilac, 3270/Sul

64001-280 - Ilhotas, Teresina, PI, Brasil

E-mail: gvmesquita@uol.com.br
Recebido em: 18 de agosto de 2008 Aprovação final: 27 de maio de 2009 